\title{
Increased skin permeation efficiency of imperatorin via charged ultradeformable lipid vesicles for transdermal delivery
}

This article was published in the following Dove Press journal: International Journal of Nanomedicine

\author{
Hongwei Lin ${ }^{1,2}$ \\ Qingchun Xie ${ }^{1,2}$ \\ Xin Huang ${ }^{1,2}$ \\ Junfeng Ban ${ }^{1,2}$ \\ Bo Wang ${ }^{1,2}$ \\ Xing $\mathrm{Wei}^{3}$ \\ Yanzhong Chen ${ }^{1,2}$ \\ Zhufen Lu',2
}

'Guangdong Provincial Key Laboratory of Advanced Drug Delivery Systems, Guangdong Pharmaceutical University, Guangzhou, People's Republic of China; ${ }^{2}$ Guangdong Provincial Engineering Center of Topical Precise Drug Delivery System, Guangdong Pharmaceutical University, Guangzhou, People's Republic of China; ${ }^{3}$ Guangdong Shennong Chinese Medicine Research Institute, Guangzhou, People's Republic of China
Correspondence: Yanzhong Chen; Zhufen Lu

Guangdong Provincial Key Laboratory of Advanced Drug Delivery Systems, Guangdong Pharmaceutical University, 280 Waihuan East Road, Guangzhou Higher Education Mega Center, Guangzhou 510006, People's Republic of China

Tel +86 $2039352678 ;+862039352506$

Fax +86203935250I

Email doctor.c@163.com;

luzhufen@163.com
Aim: The aim of this work was to develop a novel vesicular carrier, ultradeformable liposomes (UDLs), to expand the applications of the Chinese herbal medicine, imperatorin (IMP), and increase its transdermal delivery.

Methods: In this study, we prepared IMP-loaded UDLs using the thin-film hydration method and evaluated their encapsulation efficiency, vesicle deformability, skin permeation, and the amounts accumulated in different depths of the skin in vitro. The influence of different charged surfactants on the properties of the UDLs was also investigated.

Results: The results showed that the UDLs containing cationic surfactants had high entrapment efficiency $(60.32 \% \pm 2.82 \%)$, an acceptable particle size $(82.4 \pm 0.65 \mathrm{~nm})$, high elasticity, and prolonged drug release. The penetration rate of IMP in cationic-UDLs was 3.45-fold greater than that of IMP suspension, which was the highest value among the vesicular carriers. UDLs modified with cationic surfactant also showed higher fluorescence intensity in deeper regions of the epidermis. Conclusion: The results of our study suggest that cationic surfactant-modified UDLs could increase the transdermal flux, prolong the release of the drug, and serve as an effective dermal delivery system for IMP.

Keywords: ultradeformable liposomes, cationic, imperatorin, skin permeation, transdermal drug delivery

\section{Introduction}

Over the past few decades, there has been a significant increase in interest in transdermal drug delivery as a means to facilitate skin penetration of drugs. ${ }^{1}$ Transdermal drug delivery provides a number of advantages for systemic drug therapy, such as avoiding hepatic first-pass metabolism, stabilizing drug plasma levels, reducing adverse effects of gastrointestinal absorption, and improving patient compliance. ${ }^{2}$ Despite these distinct advantages of transdermal delivery, most drugs are difficult to partition into and diffuse through the skin because of the barrier function of the stratum corneum, the outermost layer of the skin epidermis. ${ }^{3}$ Various approaches, including the use of chemical permeation enhancers, ultrasound, electroporation, iontophoresis, microneedles, and laser thermal ablation have been used to increase the efficacy of transdermal pathways. ${ }^{4}$ Among several approaches, noninvasive vesicular delivery seems promising and has gained importance in transdermal drug delivery. ${ }^{5}$

Liposomes, as nanosized carriers, composed of biodegradable phospholipids, can carry lipophilic or hydrophilic drugs and facilitate their skin permeation. ${ }^{6}$ However, it has become evident that it is difficult for conventional liposomes (CLs) to penetrate deep into the skin, with a large fraction retained in upper layers of stratum corneum 
and accumulated in skin appendages. ${ }^{7}$ It has been reported that deformable liposomes, consisting of phospholipids and so-called edge activators, could be driven deep into the skin by a hydration gradient when applied nonocclusively. ${ }^{8}$ The edge activators used for the preparation of deformable liposomes are generally single-chain surfactants with a high radius of curvature, which destabilizes and increases deformability of the lipid bilayers of the vesicles. ${ }^{9}$ These new functional liposomes, called ultradeformable liposomes (UDLs), are a type of elastic vesicle, first introduced by Cevc et al. ${ }^{10}$ UDLs have the ability to squeeze themselves through intercellular sealing lipids of the stratum corneum as intact vesicles, without permanent disintegration. ${ }^{11}$ While UDLs can increase the skin penetration of transdermally administered drugs both in vitro and in vivo, ${ }^{12-14}$ the influence of surface charge modifications of UDLs on drug permeation remains unclear.

Lipid lamellae of the stratum corneum contain a high proportion of negatively charged lipids, indicating that the skin could act as a negatively charged membrane. ${ }^{15}$ It may, therefore, cause some components of the liposome bilayer to be transferred into the skin. ${ }^{16}$ Regarding the effect of the surface charge of liposomes on drug penetration, the skin permeation of drugs incorporated in positively charged liposomes was found to be higher compared with that of negatively charged liposomes. ${ }^{17-19}$ This phenomenon, indicating that positively charged liposomes remarkably enhance the penetration of drugs across the skin, probably owing to the negatively charged stratum corneum, favors the electrostatic attraction of positively charged liposomes. ${ }^{20}$ Thus, it has been proposed that the electrostatic interaction between the negatively charged skin surface and positively charged liposomes could promote drug permeation and its consequent rapid depletion by the bloodstream in vascularized sections of the skin. ${ }^{21}$ However, the effectiveness of anionic surfactants in transdermal liposomes has also been reported. ${ }^{22,23}$ The abovementioned hypothetical views evoked our interest in exploring the influence of surface modification in UDLs to improve transdermal drug absorption.

Imperatorin (IMP) is an important active compound from the Chinese herbal medicinal plant, Angelica dahurica. ${ }^{24}$ Pharmacological activities of IMP have long been recognized, including anti-inflammatory, ${ }^{25}$ analgesic, ${ }^{26}$ antibacterial, ${ }^{27}$ antitumor ${ }^{28}$ anticonvulsant, ${ }^{29}$ and anticoagulant activities. ${ }^{30}$ IMP has also been investigated as both an antihypertensive and cardioprotective agent. ${ }^{31}$ Considering the variety of pharmacological properties displayed by IMP, it is clear that the compound has the potential for development as a pharmaceutical formulation for subsequent clinical assessment. ${ }^{32}$ Because of the low bioavailability of IMP, which may be attributed to its poor absorption or extensive metabolism, ${ }^{33}$ topical application of IMP would be of great interest for its future use in clinical treatments. However, the high nonpolarity of IMP becomes an obstruction for delivering through the stratum corneum and viable epidermis, which greatly limits the percutaneous penetration of IMP.$^{34}$ Hence, it is necessary to prepare IMP-loaded UDLs for efficient transdermal delivery. The application of UDLs would simplify IMP therapy, increasing drug concentration in the skin and bioavailability, as well as improving patient compliance.

In this study, a comparison among different types of surface-charged UDLs and CLs was made to explore their potential to enhance the skin permeation of IMP. We aimed to deliver IMP into the skin by topical application using transdermal lipid vesicles and to investigate the influence of surface charge modifications on physicochemical properties and transdermal skin penetration of IMP. For this purpose, IMP-loaded lipid vesicles were prepared by the sonication method, and their physicochemical properties were characterized in terms of size, polydispersity index (PDI), zeta potential, entrapment efficiency (EE), and deformability. In vitro skin permeation and the penetration depth of different lipid vesicles were investigated. The influence of lipid vesicles on skin structure was also elucidated after the skin penetration experiments.

\section{Materials and methods Materials}

Phosphatidylcholine from soybean (90\%) was purchased from Maxim (Jiangsu, China). Cholesterol and dicetyl phosphate were purchased from Aladdin (Shanghai, China). IMP was supplied by Sunny Biotech Co., Ltd. (Shanghai, China). Stearylamine was purchased from Macklin (Shanghai, China). Polyoxyethylene (20) sorbitan monolaurate (TW20) and polyoxyethylene (80) sorbitan monolaurate (TW80) were obtained from the YOUPUHUI Pharmaceutical Co., (Shenzhen, China). All other chemicals were of analytical grade.

\section{Preparation of IMP-loaded lipid vesicles}

All lipid vesicles were prepared by a thin-film hydration method. ${ }^{35}$ Four types of ultradeformable lipid vesicles, cationic-UDLs, UDLs, anionic-UDLs, and CLs, were prepared, according to the vesicle composition presented in Table 1.

Briefly, lipid mixtures of phosphatidylcholine, cholesterol, the surfactant (stearylamine or dicetyl phosphate), and 
Table I Composition of imperatorin-loaded lipid vesicle formulations

\begin{tabular}{|c|c|c|c|c|c|c|c|}
\hline \multirow[t]{2}{*}{ Formulations } & \multicolumn{6}{|c|}{ Lipid component (mg/mL) } & \multirow[t]{2}{*}{$\overline{\text { PBS (mL) }}$} \\
\hline & Imperatorin & PC & Chol & TW20 & SA & DP & \\
\hline Cationic-UDLs & 1 & 64 & 4.26 & 11.33 & 2 & - & 1 \\
\hline UDLs & 1 & 64 & 6.26 & 11.33 & - & - & $\mathrm{I}$ \\
\hline Anionic-UDLs & I & 64 & 2.21 & 11.33 & - & 4.05 & I \\
\hline CLs & 1 & 64 & 17.6 & - & - & - & 1 \\
\hline
\end{tabular}

Abbreviations: -, component was not added in the formulation; Chol, cholesterol; CLs, conventional liposomes; DP, dicetyl phosphate; PBS, phosphate-buffered saline; PC, phosphatidylcholine; SA, stearylamine; TW20, Tween ${ }^{\circledast} 20$; UDLs, ultradeformable liposomes.

IMP were dissolved in TW20/ethanol (1\%, w/v), with TW20 playing the role of the edge activator. The organic solvent was removed by rotary evaporation (RE52-05; Shanghai Ya Rong Biochemistry Instrument Factory, Shanghai, China) under reduced pressure at $55^{\circ} \mathrm{C}$ to form a thin lipid film. The thin film was further dried under a stream of nitrogen gas for $6 \mathrm{~h}$ to remove traces of the organic solvent. The dried film was hydrated with phosphate-buffered saline (PBS, pH 7.4) for $30 \mathrm{~min}$ and then sonicated in an ice bath using a probe sonicator (JY88-IIN; Ningbo Scientz Biotechnology Co., Ltd., Zhejiang, China) for $10 \mathrm{~min}$. CLs were prepared by the same method without surfactant and edge activator. Prepared lipid vesicles were stored at $4^{\circ} \mathrm{C}$ until further analysis.

An IMP suspension was prepared by adding IMP to PBS $(\mathrm{pH} 7.4)$ at a concentration of $1.5 \mathrm{mg} / \mathrm{mL}$ and then stirring the suspension for $48 \mathrm{~h}$ to ensure that the IMP powder was completely dispersed. The concentration of IMP in the suspension was determined, and the suspension was used as a control in the skin permeation experiment.

\section{Particle size and zeta potential investigation}

The mean particle size and PDI of the prepared liposomes were determined by dynamic light scattering using a particle size and zeta-potential analyzer (Delsa Nano C; Beckman Coulter, Brea, CA, USA). Each vesicle formulation was diluted 10 -fold with filtered deionized water prior to the measurements. The zeta potential was measured by electrophoretic light scattering using the same instrument. All measurements were performed at room temperature in triplicate.

\section{Transmission electron microscopy (TEM)}

The morphology of liposomes was characterized by TEM (HT7700, Hitachi, Tokyo, Japan). One drop of each liposome vesicle preparation was placed onto a copper grid, and the excess suspension was immediately adsorbed using filter paper. The sample was then negatively stained by adding a drop of $2 \%$ phosphotungstic acid, followed by drying at room temperature. Afterward, the grid was observed using a TEM with an accelerating voltage of $120 \mathrm{kV} .{ }^{36}$

\section{EE of lipid vesicles}

The non-entrapped drug was separated from drug-loaded liposomes by dialysis. ${ }^{37}$ Drug-loaded liposomes $(0.5 \mathrm{~mL})$ were placed into a cellulose acetate dialysis tube (molecular weight cutoff 3,000; Guangzhou Qiyun Bio Technology Co., Ltd., Guangdong, China). Then, the dialysis tube was immersed in $20 \mathrm{~mL}$ of $0.5 \%$ TW 80 in a closed vessel, followed by stirring at $100 \mathrm{rpm}$ on a shaking table (THZ-8Z; Jiangsu Jintan Honghua Instrument Plant, Jiangsu, China) at $37^{\circ} \mathrm{C}$. Aliquots of the dialysate were withdrawn at certain time points and replaced with equal volumes of fresh solvent. Equilibrium was considered achieved when constant drug concentrations were obtained in dialysate samples. The concentration of IMP in the samples was determined by high-performance liquid chromatography (HPLC). The EE (\%) of the lipid vesicles was calculated according to the following equation:

$$
\mathrm{EE} \%=\frac{W_{\text {Total }}-W_{\text {Free }}}{W_{\text {Total }}} \times 100
$$

where $W_{\text {Total }}$ and $W_{\text {Free }}$ represent the weights of the total and non-encapsulated drug, respectively.

\section{Evaluation of deformability of lipid vesicles}

Deformability is a critical factor for efficient skin permeation of UDLs. The liposome vesicles were extruded through 50-nm polycarbonate membranes (LiposoFast-Basic; Avestin, Inc., Ottawa, Canada) at a constant pressure of $0.2 \mathrm{MPa} .^{38}$ The amount of vesicles that was extruded within 5 min was measured, and the vesicle size was monitored by dynamic light scattering after filtration. The deformability 
index $(D)$ of the liposome vesicles was calculated by the following equation:

$$
D=J \times\left(\frac{r_{v}}{r_{p}}\right)^{2}
$$

where $D$ is the deformability of vesicles, $J$ is the rate of penetration through the permeability membrane, $r_{v}$ is the particle size after extrusion, and $r_{p}$ is the pore diameter of the permeability membrane.

\section{In vitro skin permeation of lipid vesicles Preparation of rat skin}

The skin for permeation experiments was excised from the abdominal area of healthy male Sprague-Dawley rats weigh-

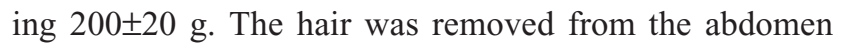
without damaging the skin with a sufficient amount of an $8 \% \mathrm{Na}_{2} \mathrm{~S}$ solution. After the rats were euthanized by cervical dislocation, the abdominal skin was surgically separated. Then, underlying fat and subcutaneous tissue were carefully removed. The full-thickness skin was washed with normal saline and stored in a polyethylene bag at $-20^{\circ} \mathrm{C} .{ }^{39}$ All animal protocols complied with the Guide for the Care and Use of Laboratory Animals of the National Institutes of Health for the care of laboratory animals, and all animal experiments were approved by the Institutional Animal Care and Use Committee of Guangdong Pharmaceutical University.

\section{Skin permeation study}

Skin permeation of the lipid vesicles was evaluated with the isolated rat skin using a Franz diffusion cell (Shanghai Kai Kai Technology Trade Co., Ltd., Shanghai, China). The available area of the diffusion cell was $3.14 \mathrm{~cm}^{2}$. The full-thickness rat skin was mounted between the donor and receptor compartments so that the stratum corneum side of the skin faced the donor chamber. The receptor compartment was filled with $18 \mathrm{~mL}$ of normal saline solution, and TW80 $(1 \%, w / w)$ was added in order to ensure the sink condition. After equilibration for $15 \mathrm{~min}$ in a water bath, $1 \mathrm{~mL}$ of an IMP-loaded vesicle formulation was applied onto the skin on the donor compartment side. The receptor compartment was maintained at $32^{\circ} \mathrm{C}$, with the solution constantly stirred at $400 \mathrm{rpm}$ during the experiment. ${ }^{21}$ A non-occlusive condition was maintained to generate a driving force for skin permeation of UDLs and to simulate an in vivo application. ${ }^{8}$ At time points of 1, 2, 4, 6, 8, 10, 12, and $24 \mathrm{~h}, 2 \mathrm{~mL}$ of the receiving medium was withdrawn and immediately replaced with an equal volume of fresh diffusion solution. Sink conditions were maintained throughout the experimental procedure. The amount of drug in the samples was analyzed by HPLC, using a C18 column $(5 \mu \mathrm{m}, 25 \mathrm{~cm} \times 4.6 \mathrm{~mm}$; Kromasil, Bohus, Sweden) with a mobile phase consisting of methanol/water (70:30). The flow rate was $1.0 \mathrm{~mL} / \mathrm{min}$ at room temperature, and the wavelength used for detection was $297 \mathrm{~nm}$. The cumulative amount of IMP permeated per unit area was calculated using the following equation: ${ }^{40}$

$$
Q_{n}=\frac{C_{n} V_{r}+\sum \frac{n-1}{i=1} C_{i} V_{s}}{A}
$$

where $Q_{n}$ is the cumulative amount of the drug permeated per unit area $\left(\mu \mathrm{g} / \mathrm{cm}^{2}\right)$ at different sampling times, $C_{n}$ is the drug concentration in the receiving medium at different sampling times, $C_{i}$ is the drug concentration in the receiving medium at the $i$ th $\left(n^{-1}\right)$ sampling time, $V_{r}$ is the volume of the receptor solution $(18 \mathrm{~mL}), V_{s}$ is the volume of the sample withdrawn ( $2 \mathrm{~mL}$ ), and $A$ is the effective permeation area of the diffusion cell $\left(3.14 \mathrm{~cm}^{2}\right)$.

The $Q_{n}$ values were plotted against time, and then the steady-state flux $\left(J_{s s}\right)$ and lag time $\left(t_{\text {lag }}\right)$ were calculated from the slope and $X$-intercept of the linear portion of the plot, respectively. The permeability coefficient $\left(K_{p}\right)$ was calculated with the following formula:

$$
K_{p}=\frac{J_{s s}}{C_{0}}
$$

where $C_{0}$ represents the initial concentration of IMP in the donor compartment.

\section{Fluorescence microscopy}

The depth of the skin penetration of lipid vesicles was evaluated by fluorescence microscopy (DMi8; Leica Microsystems, Wetzlar, Germany). Rhodamine B (RB) was entrapped in the lipid vesicles as a fluorescence marker. RB-loaded cationic-UDLs (RB-cationic-UDLs), UDLs (RB-UDLs), anionic-UDLs (RB-anionic-UDLs), and CLs (RB-CLs) were prepared with the same concentration $(0.02 \%)$ of $R B$. Diffusion studies were performed under the same conditions as the in vitro skin permeation studies. After application for $12 \mathrm{~h}$, the residual amount of the liposome formulation was removed from the diffusion area of the skin with filter paper. The skin was washed with distilled water and then incorporated into the Tissue-Tek optimum cutting temperature 
compound (O.C.T. compound, Pelco International, Redding, CA, USA) at $-20^{\circ} \mathrm{C}$ after fixation and dehydration. The frozen skin was vertically sectioned into $8-\mu \mathrm{m}$ thick slices with a cryostat microtome and mounted on positively charged adhesion microscope slides. The skin sections were optically scanned at $8-\mu \mathrm{m}$ increments without any additional staining or treatment through a $20 \times$ objective using a fluorescence microscope equipped with a filter for $\mathrm{RB}$. The images were then quantitatively analyzed for the sum of fluorescence integral optical intensities at various depths using Image-Pro Plus software. ${ }^{41,42}$

\section{Characterization of skin structure after lipid vesicle treatment}

After the skin permeation experiment, the skin was carefully washed with distilled water and blotted dry. The skin structure, mainly the lipid organization in the stratum corneum, was investigated after treatment with the different types of UDLs and suspension by differential scanning calorimetry ${ }^{43}$ and attenuated total reflectance Fourier transform infrared spectroscopy (ATR-FTIR). ${ }^{44}$ The natural untreated skin was also analyzed and used as a control. Thermal analysis of the skin was performed with a differential scanning calorimeter (DSC 4000; PerkinElmer, Waltham, MA, USA). Skin samples weighing 5-8 $\mathrm{mg}$ were compressed in a sealed aluminum pan and heated from $30^{\circ} \mathrm{C}$ to $200^{\circ} \mathrm{C}$ at a $10^{\circ} \mathrm{C} / \mathrm{min}$ increase in heating rate with a $50 \mathrm{~mL} / \mathrm{min}$ nitrogen flow. Molecular vibrations of lipid components of the skin were analyzed by ATR-FTIR. The analyzed skin samples were prepared by using the $\mathrm{KBr}$ disk method, grinding the mixture of the skin and $\mathrm{KBr}$ uniformly in a ratio of 1:100. Infrared transmission spectra were recorded using an FTIR spectrophotometer (Spectrum 100; PerkinElmer, Yokohama, Japan) in the range of $4,000-500 \mathrm{~cm}^{-1}$ at a resolution of $1 \mathrm{~cm}^{-1}$.

\section{Statistical analysis}

Data are expressed as the means \pm standard deviations of three independent experiments. Differences among groups were assessed by analysis of variance and between groups by the independent samples $t$-test, using SPSS software (SAS Institute, Cary, NC, USA). A value of $P<0.05$ was considered statistically significant.

\section{Results and discussion Preparation and characterization of IMP-loaded lipid vesicles}

The physicochemical characteristics of cationic-UDLs, UDLs, anionic-UDLs, and CLs are outlined in Table 2. The results revealed differences in the particle size $(\mathrm{nm})$, PDI, zeta potential ( $\mathrm{mV})$, and EE (\%). The particle size analysis showed that the size of all investigated lipid vesicles was in the range of $73.5 \pm 1.75$ to $93.7 \pm 1.55 \mathrm{~nm}$. The homogeneity of the size distribution, indicated by PDI values of $<0.300$, was acceptable. A small particle size of lipid vesicles is beneficial for the penetration of encapsulated drugs into deeper skin layers. ${ }^{45}$ An obvious reduction in the particle size of UDLs was observed upon adding TW20 compared with the largest particle size of CLs. The decrease in the particle size may have resulted from the curvature of the UDLs at a proper edge activator amount, which leads to a decrease in the surface energy with increasing hydrophobicity. ${ }^{46}$ Otherwise, the long alkyl chains of stearylamine and dicetyl phosphate may exhibit strong hydrophobic interactions with phosphatidylcholine, and potentially increase hydrophobic volume of the liposomes. Hence, the reductions in the particle sizes of cationic-UDLs and anionic-UDLs were smaller than that of the UDLs. ${ }^{47}$ Regarding the zeta potential, CLs and UDLs possessed a negative surface charge. The UDLs showed a negative zeta potential due to partial hydrolysis of the polyethylene oxide head groups $\left(\mathrm{CH}_{2}-\mathrm{CH}_{2}-\mathrm{O}\right)_{n}$ of TW20. ${ }^{48}$ cationic-UDLs possessed a highly positive surface charge and anionic-UDLs possessed a highly negative surface charge as a result of the intrinsic properties of the charged surfactants, stearylamine and dicetyl phosphate, respectively. The same molar ratio of stearylamine and dicetyl phosphate was used in the lipid vesicle formulations to investigate the

Table 2 Characteristics of different imperatorin-loaded lipid vesicles

\begin{tabular}{|c|c|c|c|c|}
\hline Formulations & $\begin{array}{l}\text { Particle } \\
\text { size }(\mathbf{n m})\end{array}$ & PDI & $\begin{array}{l}\text { Zeta potential } \\
(\mathrm{mV})\end{array}$ & $\begin{array}{l}\text { Entrapment } \\
\text { efficiency (\%) }\end{array}$ \\
\hline Cationic-UDLs & $82.4 \pm 0.65$ & $0.298 \pm 0.010$ & $20.22 \pm 0.96$ & $60.32 \pm 2.82$ \\
\hline UDLs & $73.5 \pm 1.75$ & $0.30 \mathrm{I} \pm 0.005$ & $-24.87 \pm 1.87$ & $57.60 \pm 2.06$ \\
\hline Anionic-UDLs & $80.2 \pm 1.14$ & $0.294 \pm 0.003$ & $-42.63 \pm 0.80$ & $54.78 \pm 1.46$ \\
\hline CLs & $93.7 \pm 1.55$ & $0.290 \pm 0.010$ & $-34.60 \pm 0.81$ & $47.23 \pm 1.71$ \\
\hline
\end{tabular}

Note: Data are presented as the means \pm standard deviations $(n=6)$.

Abbreviations: CLs, conventional liposomes; PDI, polydispersity index; UDLs, ultradeformable liposomes. 
influence of different surface charge modifications on the skin penetration. Since the long-chain carbon surfactants, stearylamine, dicetyl phosphate, and TW20, can increase the solubility of IMP in the vesicular bilayer, the EE (\%) values of cationic-UDLs, UDLs, and anionic-UDLs were significantly higher than that of the CLs. The highest EE (\%) shown by cationic-UDLs could be attributed to an increased hydrophobic area within the phosphatidylcholine bilayer for IMP by the positively charged combination. ${ }^{49}$ The TEM analysis of the lipid vesicles revealed a vesicular morphology with a spherical shape and unilamellar structure, as depicted in Figure 1. Comparison of lipid vesicles produced with different formulations indicated that the surface charge did not have a marked effect on their structures.

\section{Deformability of IMP-loaded lipid vesicles}

Deformability is a crucial characteristic of UDLs, and the permeation ability of liposomes is evaluated as the ability to squeeze through pores smaller than the liposomal diameters. The deformability of the different types of IMP-loaded lipid vesicles is shown in Figure 2. cationic-UDLs exhibited the highest deformability index (135.08 \pm 5.74$)$, while those of UDLs, anionic-UDLs, and CLs were 100.98 \pm 6.17 , $67.29 \pm 2.37$, and 55.71 \pm 1.67 , respectively. These results indicate that the addition of different surfactants had a significant effect on vesicle deformability. The high deformability of the lipid vesicles could be due to the incorporation of stearylamine, dicetyl phosphate, and TW20 into the lipid bilayer. Thus, TW20, a nonionic surfactant with a large head group and highly pliable hydrocarbon chains in its structure, afforded more flexibility to the lipid bilayer in the UDLs compared with that in the CLs. ${ }^{19}$ Therefore, the UDLs would penetrate the skin spontaneously, and the risk of the vesicles rupturing in the skin would be minimized. Stearylamine exhibited a high radius of curvature, which can destabilize and increase the deformability of the vesicle bilayer. ${ }^{50}$
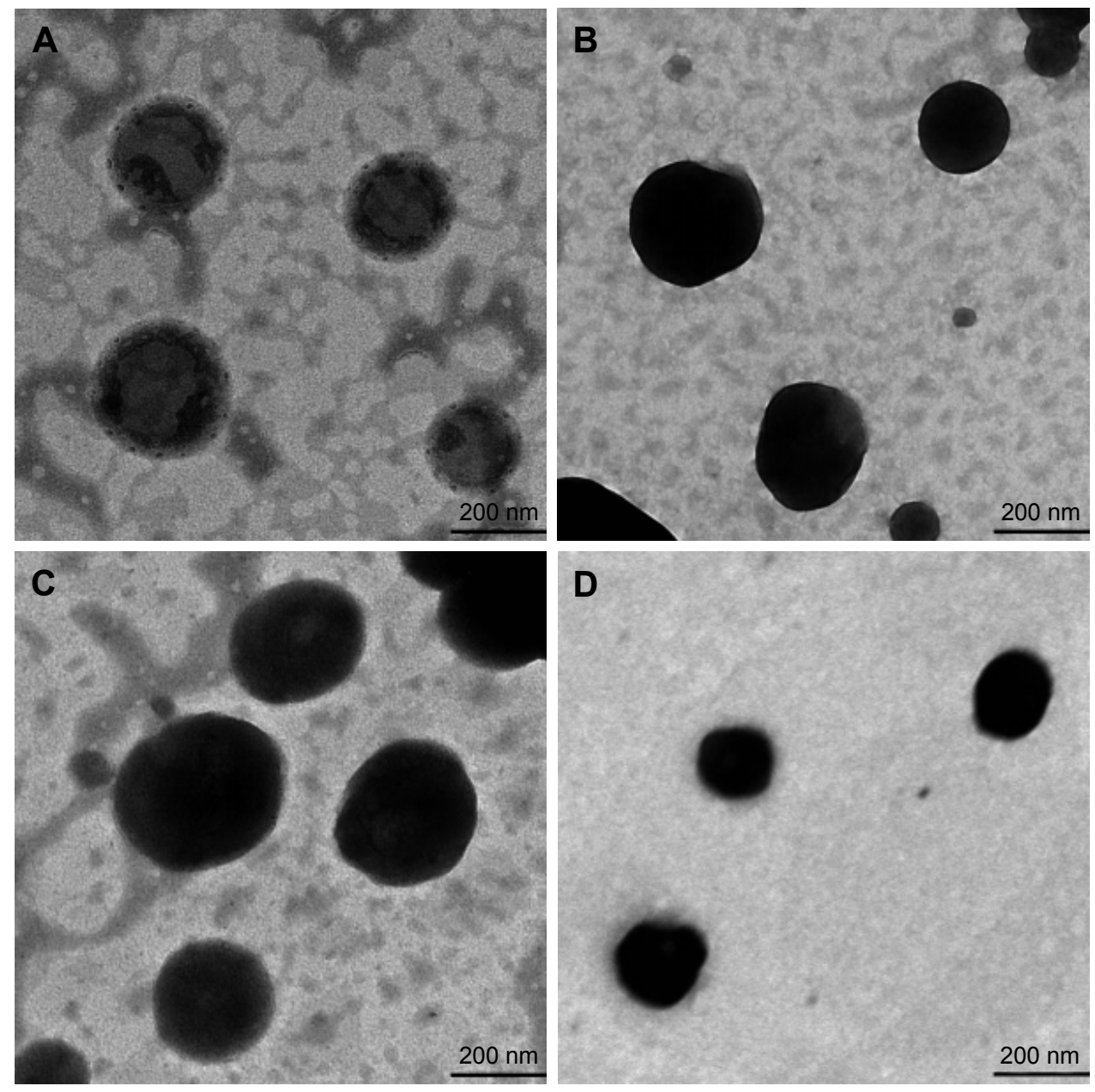

Figure I Morphology of lipid vesicles observed under transmission electron microscopy.

Notes: (A) Cationic-UDLs; (B) UDLs; (C) anionic-UDLs; (D) CLs.

Abbreviations: CLs, conventional liposomes; UDLs, ultradeformable liposomes. 


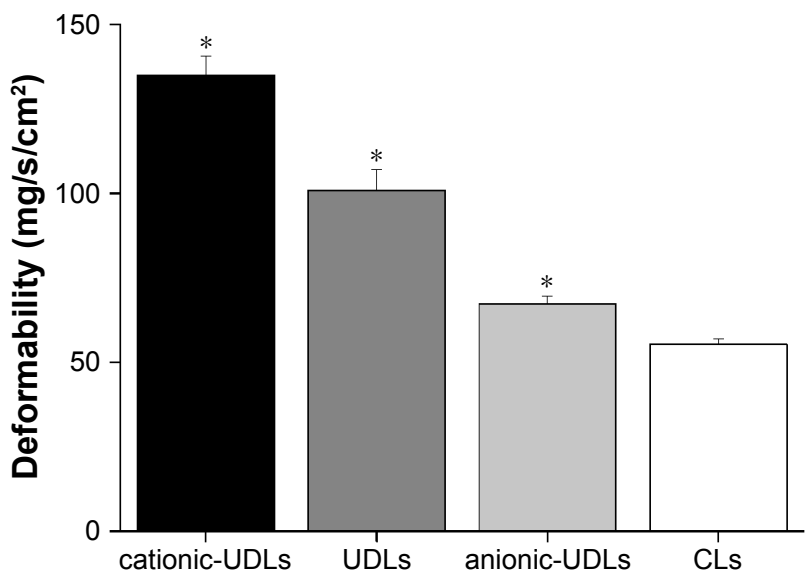

Figure 2 Deformability of different imperatorin-loaded lipid vesicles.

Notes: The deformability of imperatorin-loaded lipid vesicles was compared with that of CLs. Data are expressed as the means \pm standard deviations $(n=6) . * P<0.05$ versus CLs.

Abbreviations: CLs, conventional liposomes; UDLs, ultradeformable liposomes.

As a result of cooperation between stearylamine and TW20, the deformability of the cationic-UDLs was markedly improved. However, dicetyl phosphate has a double-long carbon chain, which is bulky in the molecular space of chemical structures, leading to less flexibility than that of hydrocarbon chains. This inflexible carbon chain prevented TW20 from incorporation into the phospholipid bilayer, which decreased the deformability of the anionic-UDLs.

\section{In vitro skin permeation of lipid vesicles}

To further improve the skin penetration of IMP, the effect of the surface charge of UDLs on drug penetration was investigated. The cumulative amounts of the different types of IMP-loaded lipid vesicles and the drug suspension, permeated per unit area, were plotted as a function of time, and their skin permeation profiles were acquired, as shown in Figure 3. Various skin permeation parameters, including $Q_{n}$ of IMP permeated within $24 \mathrm{~h}, J_{s s}, K_{p}, t_{\text {lag }}$, and the enhancement ratio of $K_{p}$, were calculated and are presented in Table 3. There were significant differences in $Q_{n}$ of IMP $(P<0.01)$ among the IMP-loaded lipid vesicles and the drug suspension after skin permeation for $24 \mathrm{~h}$. The $Q_{n}$ and $J_{s s}$ values of IMP delivered by cationic-UDLs were found to be the highest at $330.50 \pm 9.68 \mu \mathrm{g}$ and $3.54 \pm 0.09 \mu \mathrm{g} / \mathrm{cm}^{2} / \mathrm{h}$, respectively. The $Q_{n}$ and $J_{s s}$ values of the different IMP-loaded lipid vesicles and the drug suspension decreased in the order, cationicUDLs $>$ UDLs $>$ anionic-UDLs $>$ CLs $>$ suspension, which corresponded with the results of the deformability study. It was observed that the addition of stearylamine as the cationic surfactant in cationic-UDLs caused the greatest increase in $J_{s s}$, indicating a rapid achievement of the steady

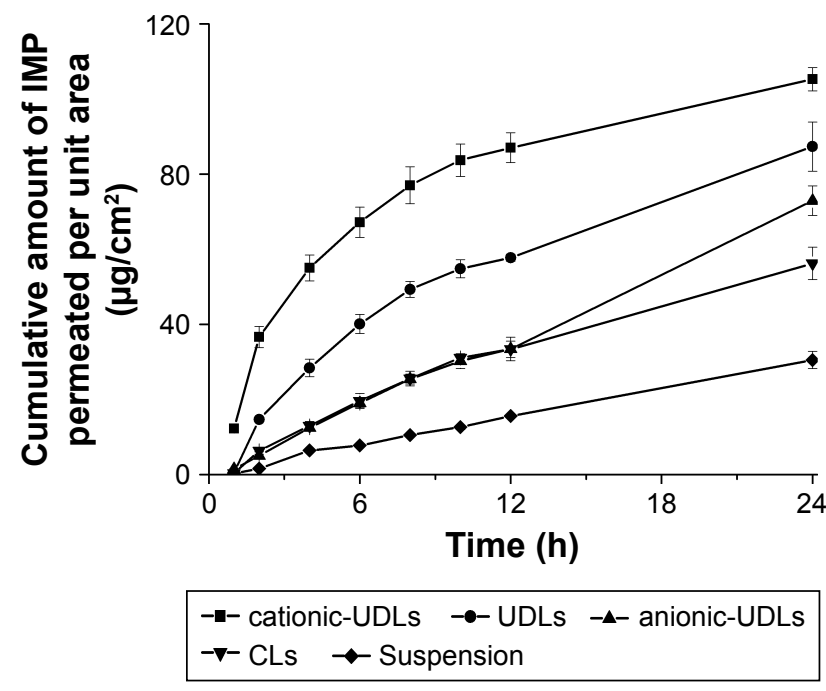

Figure 3 Rat skin permeation profiles of IMP-loaded lipid vesicles and IMP suspension, measured over $24 \mathrm{~h}$.

Note: Data are expressed as the means \pm standard deviations $(n=6)$.

Abbreviations: CLs, conventional liposomes; IMP, imperatorin; UDLs, ultradeformable liposomes.

state in skin permeation, with the shortest $t_{\text {lag }}$ of $0.61 \pm 0.04 \mathrm{~h}$. To investigate the release characteristics of the IMP-loaded lipid vesicles and the drug suspension, the skin permeation data were fitted to first-order, Higuchi, Ritger-Peppas, and Weibull equations. The most suitable equation was determined based on the coefficient of correlation $\left(R^{2}\right)$, as shown in Table 4. The final fitted release model was defined as the Higuchi equation for cationic-UDLs, UDLs, and CLs and as the first-order equation for anionic-UDLs and the IMP suspension.

The results discussed above revealed that the skin permeation of IMP could be efficiently increased with the positive charge modification of the UDLs. The IMP suspension penetrated the skin very poorly because of the large molecular weight and extremely high hydrophobicity of the drug. The enhanced skin permeation of UDLs in comparison to that of CLs was attributed to the high deformability associated with the single-chain surfactants, which form high-curvature structures, thus enabling the vesicles to deliver entrapped drugs across the skin. ${ }^{10}$ The different charged surfactants, stearylamine and dicetyl phosphate, result in swelling of the stratum corneum and interaction with intercellular lipids, which may affect the skin permeation of various drugs. ${ }^{50}$ cationic surfactants are able to extract lipids from the stratum corneum and disrupt the lipid bilayer, allowing their packing within the tissue. In contrast, anionic surfactant-treated stratum corneum is relatively brittle, possibly because of extraction of a natural moisturizing factor. ${ }^{35}$ However, lipid lamellae of the stratum corneum contain a high proportion 
Table 3 Skin permeation parameters of different formulations

\begin{tabular}{llllll}
\hline Formulations & $\begin{array}{l}\text { Cumulative amount of } \\
\text { permeation in } \mathbf{2 4} \mathbf{h}(\mu \mathrm{g})\end{array}$ & $J_{s s}\left(\mu \mathrm{g} / \mathrm{cm}^{2} / \mathbf{h}\right)$ & $K_{p}(\mathbf{c m} / \mathbf{h})$ & $\boldsymbol{t}_{\text {lag }}(\mathbf{h})$ & $\begin{array}{l}\text { Enhancement } \\
\text { ratio of } K_{p}\end{array}$ \\
\hline Cationic-UDLs & $330.50 \pm 9.68$ & $3.54 \pm 0.09$ & $2.36 \times 10^{-3}$ & $0.61 \pm 0.04$ & 2.74 \\
UDLs & $274.23 \pm 20.54$ & $3.49 \pm 0.23$ & $2.32 \times 10^{-3}$ & $1.17 \pm 0.05$ & 2.69 \\
Anionic-UDLs & $228.98 \pm 12.26$ & $3.05 \pm 0.17$ & $2.03 \times 10^{-3}$ & $1.60 \pm 0.01$ & 2.36 \\
CLs & $176.56 \pm 13.55$ & $2.35 \pm 0.18$ & $1.56 \times 10^{-3}$ & $1.41 \pm 0.02$ & 1.81 \\
Suspension & $95.85 \pm 7.21$ & $1.29 \pm 0.10$ & $0.86 \times 10^{-3}$ & $1.63 \pm 0.07$ & 1 \\
\hline
\end{tabular}

Note: Except for the $K_{p}$ and enhancement ratio, data are presented as the means \pm standard deviations $(n=6)$.

Abbreviations: $C L s$, conventional liposomes; $J_{s s}$, steady-state flux; $K_{p}$, permeability coefficient; $t_{\text {lag }}$ lag time; UDLs, ultradeformable liposomes.

of negatively charged lipids, which may strengthen the interaction between cationic-UDLs and intercellular lipids. ${ }^{20}$ Therefore, the different charged surfactant increased the skin permeability of the UDLs with the positive charge modification. The release characteristics of IMP penetrated with cationic-UDLs, UDLs, and CLs, which fitted well with the Higuchi equation model, showed the sustained release profile. The lipid vesicles could act as reservoir systems for continuous delivery of IMP, while the anionic-UDLs deviated from the first-order model of drug release behavior, following the diffusion-controlled characteristic.

\section{Skin penetration of a fluorescence marker (RB) entrapped in lipid vesicles}

The distribution of IMP-loaded lipid vesicles during skin penetration was investigated by detecting the accumulation of a fluorescence marker at different depths in the skin. The rat skin was treated with RB-cationic-UDLs, RB-UDLs, RB-anionic-UDLs, and RB-CLs for $12 \mathrm{~h}$. The fluorescence images of the skin at various depths are shown in Figure 4. Based on the photomicrographs taken from 0 to $48 \mu \mathrm{m}$ of the skin depth, RB-anionic-UDLs showed the highest fluorescence compared with that of the other lipid vesicles, while there was a small difference in the fluorescence of RB-cationic-UDLs and RB-UDLs in the same range of skin depth. RB-CLs showed the lowest fluorescence at every increment of the skin depth. For further quantitative analysis of RB penetration, the fluorescence intensity was plotted against the corresponding skin depth (Figure 5). Based on the results, the maximum fluorescence intensity of the RB-anionic-UDLs, 3,706.48 \pm 366.76 AU (arbitrary units), was observed at the $32-\mu \mathrm{m}$ skin depth. Meanwhile,

Table 4 Fitting equations for in vitro skin permeation of different formulations

\begin{tabular}{|c|c|c|c|}
\hline Formulations & Release model & Fitting equation & $R^{2}$ \\
\hline \multirow[t]{4}{*}{ Cationic-UDLs } & First-order equation & $\operatorname{Ln}(I-Q)=-0.0175 t-0.1402$ & 0.8101 \\
\hline & Higuchi equation & $Q=0.0857 t^{1 / 2}+0.0153$ & 0.9139 \\
\hline & Ritger-Peppas equation & $\operatorname{Ln} Q=0.6397 / n t-2.6844$ & 0.8659 \\
\hline & Weibull equation & $\operatorname{Ln}(\operatorname{Ln}(I /(I-Q)))=0.7068 \ln t-2.6618$ & 0.9041 \\
\hline \multirow[t]{4}{*}{ UDLs } & First-order equation & $\operatorname{Ln}(I-Q)=-0.0148 t-0.0388$ & 0.9308 \\
\hline & Higuchi equation & $Q=0.076 \mid t^{1 / 2}-0.0567$ & 0.9848 \\
\hline & Ritger-Peppas equation & $\operatorname{Ln} Q=|.4| 26 \ln t-4.9242$ & 0.7818 \\
\hline & Weibull equation & $\operatorname{Ln}(\operatorname{Ln}(I /(I-Q)))=I .465 \ln t-4.9356$ & 0.7972 \\
\hline \multirow[t]{4}{*}{ Anionic-UDLs } & First-order equation & $\operatorname{Ln}(I-Q)=-0.0119 t+0.0078$ & 0.9946 \\
\hline & Higuchi equation & $Q=0.0602 t^{1 / 2}-0.0748$ & 0.9451 \\
\hline & Ritger-Peppas equation & $\operatorname{Ln} Q=1.189 \mid \ln t-5.0133$ & 0.9778 \\
\hline & Weibull equation & $\operatorname{Ln}(\operatorname{Ln}(I /(I-Q)))=1.2262 \ln t-5.0323$ & 0.9847 \\
\hline \multirow[t]{4}{*}{ CLs } & First-order equation & $\operatorname{Ln}(I-Q)=-0.0094 t-0.0096$ & 0.9754 \\
\hline & Higuchi equation & $Q=0.0508 t^{1 / 2}-0.0521$ & 0.9973 \\
\hline & Ritger-Peppas equation & $\operatorname{Ln} Q=|.457| \ln t-5.6055$ & 0.8506 \\
\hline & Weibull equation & $\operatorname{Ln}(\operatorname{Ln}(I /(I-Q)))=1.489 \mid \ln t-5.6175$ & 0.8591 \\
\hline \multirow[t]{4}{*}{ Suspension } & First-order equation & $\operatorname{Ln}(I-Q)=-0.0044 t+0.0013$ & 0.9953 \\
\hline & Higuchi equation & $Q=0.0243 t^{1 / 2}-0.0302$ & 0.9653 \\
\hline & Ritger-Peppas equation & $\operatorname{Ln} Q=I .429 \operatorname{lnt}-6.464 \mathrm{I}$ & 0.9386 \\
\hline & Weibull equation & $\operatorname{Ln}(\operatorname{Ln}(I /(I-Q)))=I .443 I \ln t-6.47 \mid 2$ & $0.94 I I$ \\
\hline
\end{tabular}

Abbreviations: CLs, conventional liposomes; UDLs, ultradeformable liposomes. 

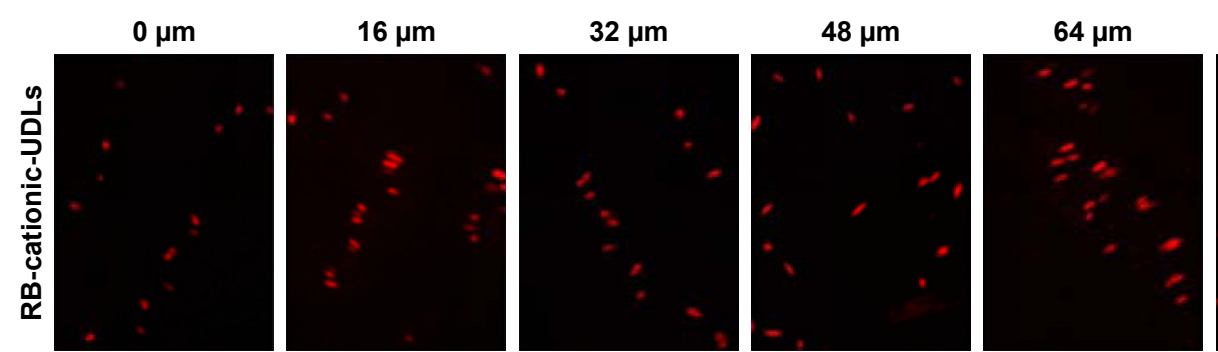

$80 \mu \mathrm{m}$
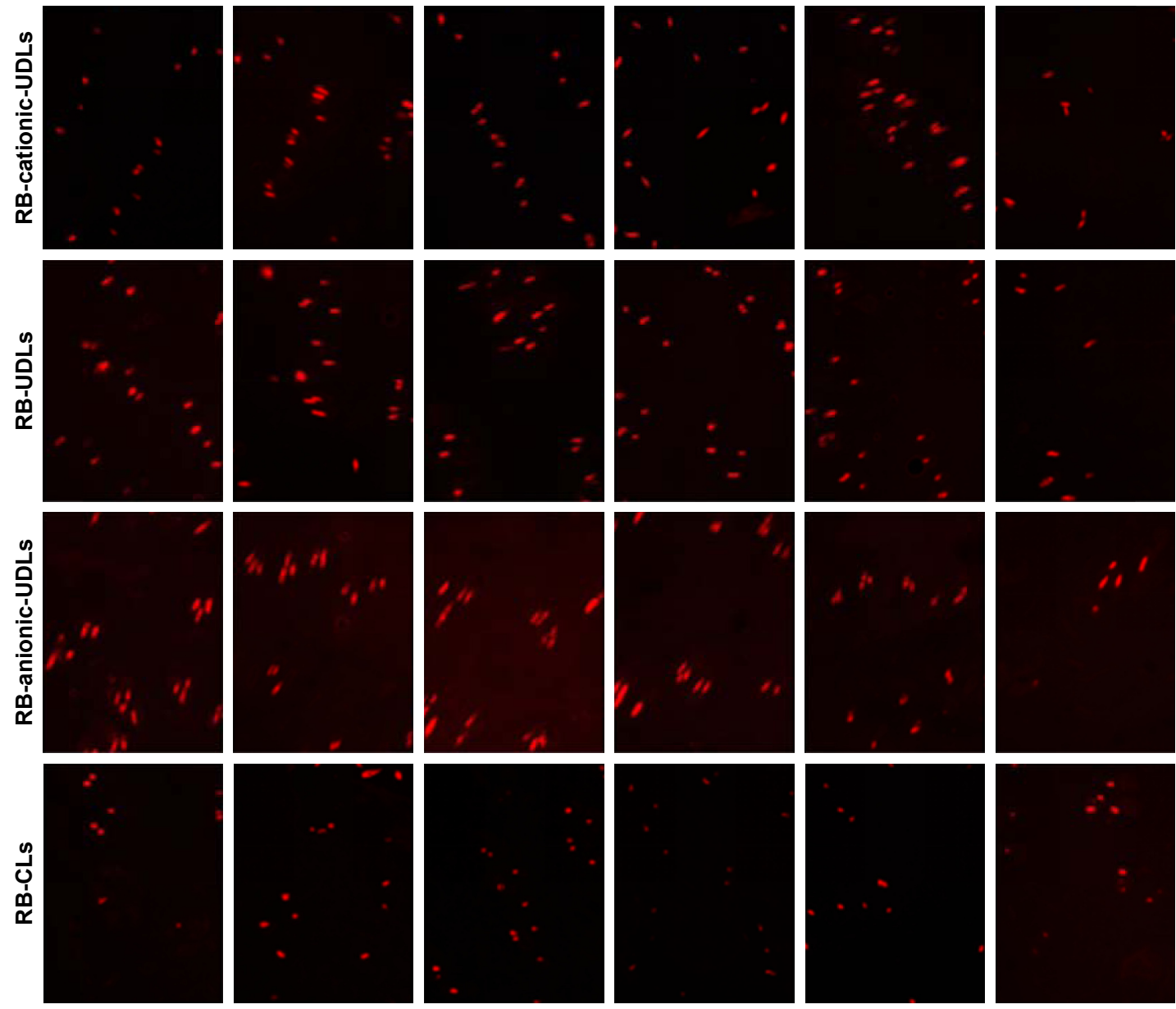

Figure 4 Fluorescence microscopy photomicrographs taken at different depths of the rat skin after $12 \mathrm{~h}$ treatment with RB-loaded lipid vesicles.

Note: Representative images are shown at $20 \times$ magnification.

Abbreviations: CLs, conventional liposomes; RB, rhodamine B; UDLs, ultradeformable liposomes.

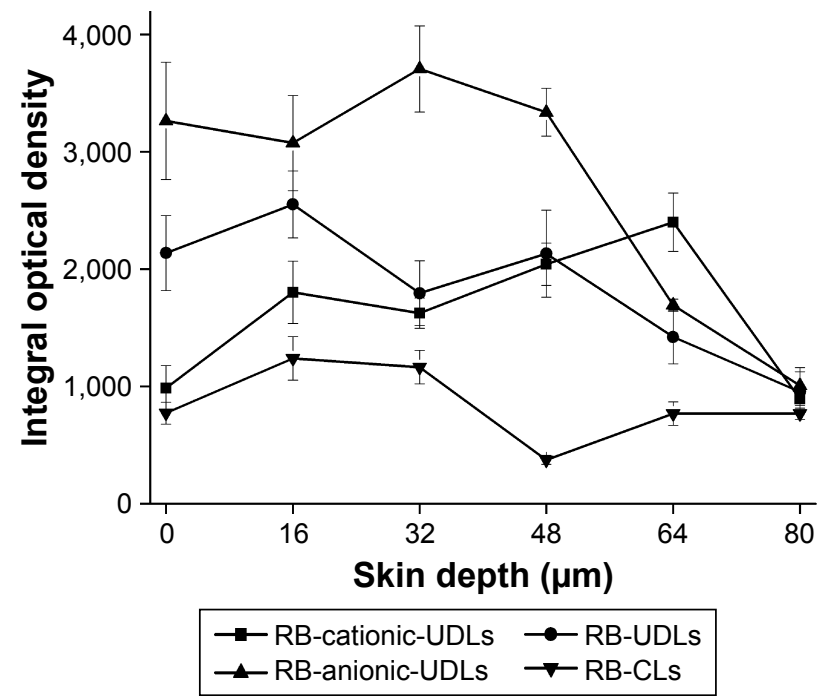

Figure $\mathbf{5}$ Fluorescence intensity versus skin permeation depth profiles of RB-loaded lipid vesicles.

Note: Data are expressed as the means \pm standard deviations $(n=6)$.

Abbreviations: CLs, conventional liposomes; RB, rhodamine B; UDLs, ultradeformable liposomes. the highest fluorescence intensity of the RB-cationic-UDLs, $2,399.97 \pm 248.62 \mathrm{AU}$, was observed at a skin depth of $64 \mu \mathrm{m}$. The fluorescence intensity of RB-cationic-UDLs deeper in the skin corresponded to improved skin permeation, rather than retention in the stratum corneum and the shallow epidermis layer, as observed with RB-UDLs and RB-anionic-UDLs. The penetration and accumulation in deeper epidermis areas were possibly attributed to the electrostatic interaction between the negatively charged epidermis layer and the cationic-UDLs, regarded as promoting transdermal absorption. ${ }^{21}$

\section{Influence of lipid vesicles on skin structure}

The influence of treatment with the vesicle formulations for $24 \mathrm{~h}$ on the organization of intercellular lipids in the skin was investigated using FTIR spectra and DSC thermograms (Figure 6). The ability of the lipid vesicles to penetrate into intercellular regions, disordering and fluidizing the intercellular lipid lattices in the stratum corneum, might be related 

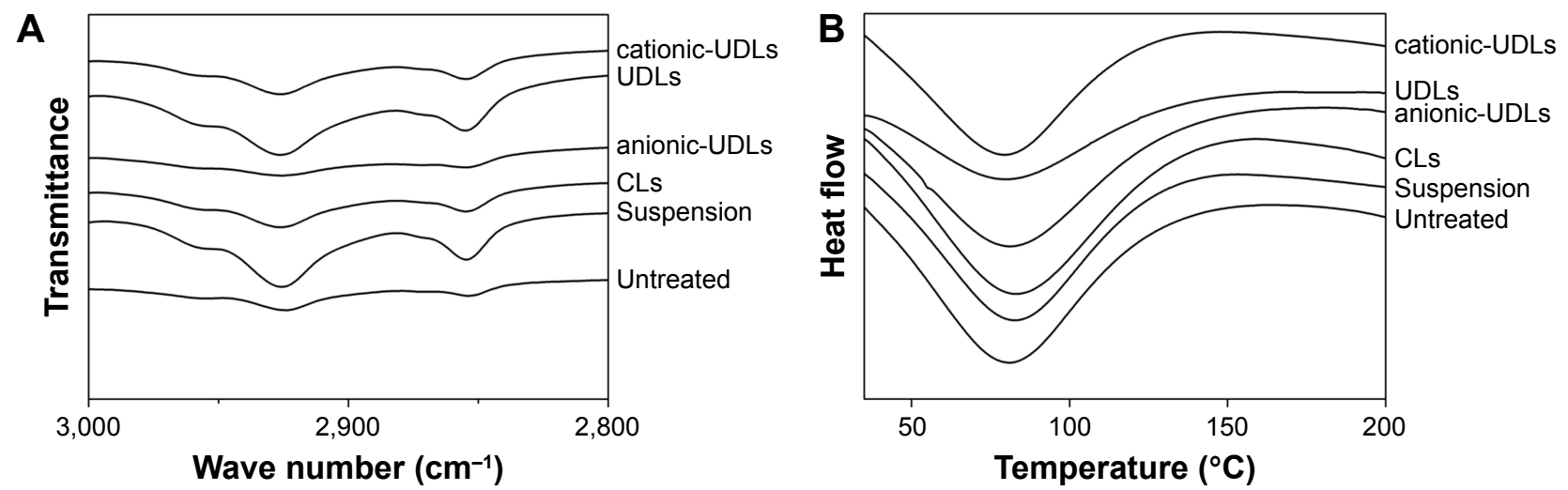

Figure 6 FTIR spectra (A) and DSC thermograms (B) of the rat skin after treatment with imperatorin-loaded lipid vesicles and an imperatorin suspension. Abbreviations: CLs, conventional liposomes; DSC, differential scanning calorimetry; FTIR, Fourier transform infrared spectroscopy; UDLs, ultradeformable liposomes.

to the enhancing effect of the transdermal permeation surfactants. ${ }^{44}$ The FTIR peaks from the asymmetric and symmetric stretching vibrations of $\mathrm{CH}_{2}$ at around 2,920 and 2,850 $\mathrm{cm}^{-1}$, respectively, were used for the evaluation of lipid acyl chains. The FTIR spectra of the natural untreated skin showed peaks at 2,924.3 and 2,853.9 $\mathrm{cm}^{-1}$, which were assigned to asymmetric and symmetric stretching vibrations of $\mathrm{CH}_{2}$, respectively. Severe structural changes in the skin would be revealed by a significant shift of the $\mathrm{CH}_{2}$ stretching vibrations. However, the FTIR spectra of the skin treated with cationic-UDLs, UDLs, anionic-UDLs, CLs, and suspension showed only slight changes in the structure of the skin, with the peaks shifting from $2,924.3 \mathrm{~cm}^{-1}$ to $2,926.2,2,926.1,2,925.5,2,926.1$, and $2,925.7 \mathrm{~cm}^{-1}$ and from $2,853.9 \mathrm{~cm}^{-1}$ to $2,854.8,2,855,2854.7$, $2,854.7$, and $2,854.6 \mathrm{~cm}^{-1}$, respectively. Meanwhile, the DSC thermograms also displayed peak shifts. The natural untreated skin used as a control showed endothermic transitions at $78.8^{\circ} \mathrm{C}$ for the transformation from a lamellar to disordered state in the lipid structure. ${ }^{43}$ The DSC thermograms of the skin treated with cationic-UDLs, UDLs, anionic-UDLs, CLs, and suspension showed endothermic transitions at 79.4, 79.8, $80.9,82.9$, and $82.6^{\circ} \mathrm{C}$, respectively These results revealed the different changes in the lipid enthalpy of the stratum corneum lipid-related transitions in the skin. The results of the FTIR and DSC analyses demonstrated that the fluidity of the stratum corneum lipids was disrupted by the vesicle formulations without serious structural changes after the skin was treated with the lipid vesicles. These results suggest that the interruption of the stratum corneum lipids by the lipid vesicles improved the skin permeation of IMP.

\section{Conclusion}

In this study, different charge-modified UDLs and CLs were prepared, and the physicochemical characteristics of the vesicles, as well as their skin permeability were evaluated.
With the positive charge modification of the UDLs, the skin permeation of IMP could be efficiently increased compared with that of the other lipid vesicles. cationic-UDLs delivered the highest amount of IMP across the skin into deeper regions of the epidermis. Stratum corneum lipids could be disrupted after the treatment with lipid vesicles. Consequently, IMPloaded cationic-UDLs could be used as transdermal lipid vesicles to achieve a desired therapeutic effect for treating skin inflammation or bacterial infection at a lower dose of the compound. Our results provide useful fundamental information for further development of IMP or other drug delivery tools for cutaneous penetration. The in vivo pharmacokinetics of IMP-loaded cationic-UDLs applied to the rat skin model is now available for future investigations.

\section{Acknowledgments}

This work was supported by the R \& D Team for Formulation Innovation (grant number 2015KCXTD026) and the Innovation and Strong School Project of Guangdong Pharmaceutical University (grant number 2015CXQX150).

\section{Disclosure}

The authors report no conflicts of interest in this work.

\section{References}

1. Sinico C, Fadda AM. Vesicular carriers for dermal drug delivery. Expert Opin Drug Deliv. 2009;6(8):813-825.

2. Chen J, Lu WL, Gu W, Lu SS, Chen ZP, Cai BC. Skin permeation behavior of elastic liposomes: role of formulation ingredients. Expert Opin Drug Deliv. 2013;10(6):845-856.

3. Proksch E, Brandner JM, Jensen JM. The skin: an indispensable barrier. Exp Dermatol. 2008;17(12):1063-1072.

4. Schoellhammer CM, Blankschtein D, Langer R. Skin permeabilization for transdermal drug delivery: recent advances and future prospects. Expert Opin Drug Deliv. 2014;11(3):393-407.

5. Priyanka K, Singh S. A review on skin targeted delivery of bioactives as ultradeformable vesicles: overcoming the penetration problem. Curr Drug Targets. 2014;15(2):184-198.

6. Schmid MH, Korting HC. Therapeutic progress with topical liposome drugs for skin disease. Adv Drug Deliver Rev. 1996;18(3):335-342. 
7. Verma DD, Verma S, Blume G, Fahr A. Liposomes increase skin penetration of entrapped and non-entrapped hydrophilic substances into human skin: a skin penetration and confocal laser scanning microscopy study. Eur J Pharm Biopharm. 2003;55(3):271-277.

8. Cevc G, Blume G. Lipid vesicles penetrate into intact skin owing to the transdermal osmotic gradients and hydration force. Biochim Biophys Acta. 1992;1104(1):226-232.

9. Cevc G, Blume G, Sch Tzlein A, Gebauer D, Paul A. The skin: a pathway for systemic treatment with patches and lipid-based agent carriers. Adv Drug Deliver Rev. 1996;18(3):349-378.

10. Cevc G, Schäitzlein A, Blume G. Transdermal drug carriers: basic properties, optimization and transfer efficiency in the case of epicutaneously applied peptides. J Control Release. 1995;36(1-2):3-16.

11. Benson HA. Transfersomes for transdermal drug delivery. Expert Opin Drug Deliv. 2006;3(6):727-737.

12. Elsayed MM, Abdallah OY, Naggar VF, Khalafallah NM. Deformable liposomes and ethosomes as carriers for skin delivery of ketotifen. Pharmazie. 2007;62(2):133-137.

13. Li S, Qiu Y, Zhang S, Gao Y. Enhanced transdermal delivery of 18betaglycyrrhetic acid via elastic vesicles: in vitro and in vivo evaluation. Drug Dev Ind Pharm. 2012;38(7):855-865.

14. Goindi S, Kumar G, Kumar N, Kaur A. Development of novel elastic vesicle-based topical formulation of cetirizine dihydrochloride for treatment of atopic dermatitis. AAPS Pharm Sci Tech. 2013; 14(4):1284-1293.

15. Burnette RR, Ongpipattanakul B. Characterization of the permselective properties of excised human skin during iontophoresis. J Pharm Sci. 1987;76(10):765-773.

16. Gonzalez-Rodriguez ML, Rabasco AM. Charged liposomes as carriers to enhance the permeation through the skin. Expert Opin Drug Deliv. 2011;8(7):857-871.

17. Katahira N, Murakami T, Kugai S, Yata N, Takano M. Enhancement of topical delivery of a lipophilic drug from charged multilamellar liposomes. J Drug Target. 1999;6(6):405-414.

18. Manosroi A, Podjanasoonthon K, Manosroi J. Development of novel topical tranexamic acid liposome formulations. Int J Pharm. 2002; 235(1-2):61-70.

19. Song YK, Kim CK. Topical delivery of low-molecular-weight heparin with surface-charged flexible liposomes. Biomaterials. 2006; 27(2):271-280.

20. Kitagawa S, Kasamaki M. Enhanced delivery of retinoic acid to skin by cationic liposomes. Chem Pharm Bull (Tokyo). 2006;54(2):242-244.

21. Dragicevic-Curic N, Grafe S, Gitter B, Winter S, Fahr A. Surface charged temoporfin-loaded flexible vesicles: in vitro skin penetration studies and stability. Int J Pharm. 2010;384(1-2):100-108.

22. Ogiso T, Yamaguchi T, Iwaki M, Tanino T, Miyake Y. Effect of positively and negatively charged liposomes on skin permeation of drugs. J Drug Target. 2001;9(1):49-59.

23. Gillet A, Compere P, Lecomte F, et al. Liposome surface charge influence on skin penetration behaviour. Int J Pharm. 2011;411(1-2) 223-231.

24. Li H, Chen F. Preparative isolation and purification of bergapten and imperatorin from the medicinal plant Cnidium monnieri using high-speed counter-current chromatography by stepwise increasing the flow-rate of the mobile phase. J Chromatogr A. 2004;1061(1): 51-54.

25. Garcia-Argaez AN, Apan T, Delgado HP, Velazquez G, MartinezVazquez M. Anti-inflammatory activity of coumarins from Decatropis bicolor on TPA ear mice model. Planta Med. 2000;66(3):279-281.

26. Zhang J, Yang G, Hu Z, He L, Li H. LC-ESI-MS Determination of imperatorin in rat plasma after oral administration and total furocoumarins of radix angelica dahuricae and its application to a Pharmacokinetic Study. Chromatographia. 2009;69(9-10):859-864.

27. Stavri M, Gibbons S. The antimycobacterial constituents of dill (Anethum graveolens). Phytother Res. 2005;19(11):938-941.

28. Kawaii S, Tomono Y, Ogawa K, et al. Antiproliferative effect of isopentenylated coumarins on several cancer cell lines. Anticancer Res. 2001 21(3B):1905-1911.
29. Rosselli S, Maggio A, Bellone G, et al. Antibacterial and anticoagulant activities of coumarins isolated from the flowers of Magydaris tomentosa. Planta Med. 2007;73(2):116-120.

30. Luszczki JJ, Glowniak K, Czuczwar SJ. Time-course and doseresponse relationships of imperatorin in the mouse maximal electroshock seizure threshold model. Neurosci Res. 2007;59(1):18-22.

31. Zhang Y, Cao Y, Wang Q, Zheng L, Zhang J, He L. A potential calcium antagonist and its antihypertensive effects. Fitoterapia. 2011; 82(7):988-996.

32. Koziol E, Skalicka-Wozniak K. Imperatorin-pharmacological meaning and analytical clues: profound investigation. Phytochem Rev. 2016; 15:627-649.

33. Zhao G, Peng C, Du W, Wang S. Simultaneous determination of imperatorin and its metabolites in vitro and in vivo by a GC-MS method: application to a bioavailability and protein binding ability study in rat plasma. Biomed Chromatogr. 2014;28(7):947-956.

34. Foldvari M. Non-invasive administration of drugs through the skin: challenges in delivery system design. Pharm Sci Technol Today. 2000; 3(12):417-425.

35. Duangjit S, Pamornpathomkul B, Opanasopit P, et al. Role of the charge, carbon chain length, and content of surfactant on the skin penetration of meloxicam-loaded liposomes. Int J Nanomed. 2014;9:2005-2017.

36. Belletti D, Vandelli MA, Tonelli M, et al. Functionalization of liposomes: microscopical methods for preformulative screening. J Liposome Res. 2015;25(2):150-156.

37. Maestrelli F, González-Rodríguez ML, Rabasco AM, Ghelardini C, Mura P. New "drug-in cyclodextrin-in deformable liposomes" formulations to improve the therapeutic efficacy of local anaesthetics. Int J Pharm. 2010;395(1-2):222-231.

38. Montanari J, Perez AP, Di Salvo F, et al. Photodynamic ultradeformable liposomes: design and characterization. Int J Pharm. 2007; 330(1-2):183-194.

39. Lv Q, Yu A, Xi Y, et al. Development and evaluation of penciclovirloaded solid lipid nanoparticles for topical delivery. Int J Pharm. 2009; 372(1-2):191-198.

40. Zeb A, Qureshi OS, Kim HS, Cha JH, Kim HS, Kim JK. Improved skin permeation of methotrexate via nanosized ultradeformable liposomes. Int J Nanomedicine. 2016;11:3813-3824.

41. Lopes LB, Vandewall H, Li HT, et al. Topical delivery of lycopene using microemulsions: enhanced skin penetration and tissue antioxidant activity. J Pharm Sci. 2010;99(3):1346-1357.

42. Ascenso A, Salgado A, Euleterio C, et al. In vitro and in vivo topical delivery studies of tretinoin-loaded ultradeformable vesicles. Eur J Pharm Biopharm. 2014;88(1):48-55.

43. Cui LL, Liang YY, Dong FJ, Ma L, Tu Y. Structure of rat skin after application of electret characterized by DSC. J Phys Conf Ser. 2011; 301(1):12027.

44. Obata Y, Utsumi S, Watanabe H, et al. Infrared spectroscopic study of lipid interaction in stratum corneum treated with transdermal absorption enhancers. Int J Pharm. 2010;389(1-2):18-23.

45. Verma DD, Verma S, Blume G, Fahr A. Particle size of liposomes influences dermal delivery of substances into skin. Int J Pharm. 2003; 258(1-2):141-151.

46. El Zaafarany GM, Awad GA, Holayel SM, Mortada ND. Role of edge activators and surface charge in developing ultradeformable vesicles with enhanced skin delivery. Int J Pharm. 2010;397(1-2):164-172.

47. Ali MH, Moghaddam B, Kirby DJ, Mohammed AR, Perrie Y. The role of lipid geometry in designing liposomes for the solubilisation of poorly water soluble drugs. Int J Pharm. 2013;453(1):225-232.

48. Tasi LM, Liu DZ, Chen WY. Microcalorimetric investigation of the interaction of polysorbate surfactants with unilamellar phosphatidylcholines liposomes. Colloid Surf A. 2003;213(1):7-14.

49. Dan N. Lipid tail chain asymmetry and the strength of membraneinduced interactions between membrane proteins. Biochim Biophys Acta. 2007;1768(10):2393-2399.

50. Elsayed MM, Abdallah OY, Naggar VF, Khalafallah NM. Lipid vesicles for skin delivery of drugs: reviewing three decades of research. Int $J$ Pharm. 2007;332(1-2):1-16. 


\section{Publish your work in this journal}

The International Journal of Nanomedicine is an international, peerreviewed journal focusing on the application of nanotechnology in diagnostics, therapeutics, and drug delivery systems throughout the biomedical field. This journal is indexed on PubMed Central, MedLine, CAS, SciSearch $\AA$, Current Contents $\AA /$ Clinical Medicine,

Journal Citation Reports/Science Edition, EMBase, Scopus and the Elsevier Bibliographic databases. The manuscript management system is completely online and includes a very quick and fair peer-review system, which is all easy to use. Visit http://www.dovepress.com/ testimonials.php to read real quotes from published authors.

Submit your manuscript here: http://www.dovepress.com/international-journal-of-nanomedicine-journal 